\title{
The Slow Merger of Massive Stars: Merger Types and Post-Merger Evolution
}

\author{
N. Ivanova \\ Department of Physics \& Astronomy, Northwestern University, \\ 2145 Sheridan Rd, Evanston, IL 60208, USA
}

Ph. Podsiadlowski

Department of Astrophysics, Oxford University, Oxford OX1 3RH, UK

\begin{abstract}
We study the slow merger of two massive stars inside a common envelope. The initial close binary system consists of a massive red supergiant and a main-sequence companion of a few solar masses. The merger product is a massive supergiant with an interior structure (core mass and composition profile) which is significantly different from that of a single supergiant that has evolved in isolation. Using a parameterized approach for the stream-core interaction, we modelled the merger phase and have identified three qualitatively different merger types: quiet, moderate and explosive mergers, where the differences are caused by the different response of the He burning shell. In the last two scenarios, the post-merger He abundance in the envelope is found to be substantially increased, but significant s-processing is mainly expected in the case of an explosive merger scenario. The subsequent evolution of the merger product up to the supernova stage is also discussed.
\end{abstract}

\section{Introduction}

The majority of stars are members of binary or multiple systems, and up to $50 \%$ of binaries are interacting binaries at some stage during their evolution. One of the most important evolutionary interactions for an initially close binary is the spiral-in of the two binary components inside a common envelope (CE), which is not in synchronous rotation with the embedded binary (Iben \& Livio 1993). The outcome of this evolutionary phase is either that the binary survives to produce a short-period binary, or that the binary components merge completely to form a rapidly rotating single star. In the latter case, there will be a point during the spiral-in, when the secondary starts to overfill its Roche lobe inside the common envelope and starts to transfer mass to the core of the primary, ultimately leading to the merger of the secondary with the core of the primary. We refer to this process as a slow merger since it proceeds on a timescale which is much longer than the dynamical timescale of the surrounding region of the CE. This mass transfer changes the chemical composition profile of the primary, its angular velocity profile and, in the case where the stream emanating from the secondary penetrates deep into the dense core of the primary, it can erode part 
of the core. If the resulting single star is sufficiently massive, it will continue its evolution until it explodes as a core-collapse supernova. The immediate supernova progenitor will be substantially different because of the merger, which may also affect the supernova explosion.

In this paper we present detailed models for the complete slow merger of a massive binary within a common envelope. In Section 2 we describe how we model the merger using a stellar evolution code and a parameterized model for the core-stream interaction. In Section 3 we discuss some of the results of the simulations, the response of the primary core to the penetration of hydrogen-rich material and other key factors affecting the merger results. We show that generally three different merger types can be identified and discuss their differences. We also address the question of s-processing during or after the merger.

\section{Numerical method}

Modelling the merger of two stars requires consideration of several additional aspects not generally considered in the evolution of a single star.

The first is the inclusion of the evolution of the binary, i.e. one has to follow the evolution of the orbital separation which involves tracing the orbital angular momentum lost with a stellar wind and the orbital shrinkage due to tidal and viscous dissipation, the change of the orbital angular momentum due to the mass exchange between the envelope and the secondary (accretion) and between the secondary and the primary core (mass transfer).

A second aspect includes the effects due to the presence of the secondary inside the primary envelope and the resulting response of the envelope. This includes the change of the gravitational field affecting the primary envelope and the presence of additional energy sources due to the luminosity generated by the accretion from the envelope on to the secondary and the frictional luminosity due to the differential rotation of the common envelope (see Meyer \& Meyer-Hofmeister 1979; Hjellming \& Taam 1991; Ivanova, Podsiadlowski \& Spruit 2000).

The next important problem is related to the phase where the secondary overfills its Roche lobe and transfers hydrogen-rich material to the core of the primary. In particular, this requires the self-consistent determination of the mass-loss rate from the embedded secondary into the common envelope. How deep the flow can penetrate into the core of the primary also depends on the density and the entropy of the secondary material which is altered by shocks with the ambient medium (Ivanova, Podsiadlowski, \& Spruit 2002, IPS).

A simplified scenario for the spiral-in which takes all of these effects into account for the primary can be obtained using a modified standard stellar evolution code. The deposition of hydrogen-rich material in the core of the primary is modelled using the prescription developed in IPS which is based on an estimate of the penetration depth using a modified Bernoulli integral, which takes into account the change of the entropy of the stream material. The entropy change in the post-shocked material compared to the initial state for a parameterized stellar structure is calculated according to $K_{\mathrm{S}}=1+k \cdot \eta_{\rho}^{\gamma-1}\left(M_{\text {int }} / M_{\text {ext }}\right)^{2}$. Here $k$ is an entropy change coefficient that depends on the stellar structure, its value is varied in the range of $0-0.45 ; \eta_{\rho}$ is the ratio of the stream density to the 
density of the ambient matter; $M_{\text {int }}$ and $M_{\text {ext }}$ are the internal and external Mach numbers of the flow; $\gamma$ is the adiabatic index of the stream material $(\gamma=5 / 3)$.

In addition, the calculation of nuclear reactions has to be modified: compared to the standard evolutionary situations in a single star, where the nuclear burning proceeds in regions which are either not mixed or are mixed very efficiently, the nuclear burning during the merging phase occurs in a regime where the nucleosynthesis and the mixing take place on comparable timescales. In this case the characteristic timescale of the hot CNO cycle can be comparable to the timescale of the convective mixing. This means that the change of the chemical composition in each layer due to the nuclear transformation and due to the mixing with other layers are of the same order and that therefore they need to be considered together when solving the nuclear reaction network. A method which can properly treat the nucleosynthesis in this environment has been developed by Cannon (1993) for calculations of Thorne-Żytkow objects. For our merger simulations we use this method directly in the evolutionary code for stages starting with the merging itself up to the end of the evolution or at least up to the moment when the post-merging chemical composition is uniformly mixed over convective zones in the star, well after the merger has been completed.

As the basic model for the merger calculations we considered a binary consisting of an $18 M_{\odot}$ primary and a secondary of $2 M_{\odot}$ or $1 M_{\odot}$. At the start of the spiral-in, the primary has been evolved to the point just before carbon core ignition and has a core of about $6.8 M_{\odot}$ (and a He-exhausted core of about $4.1 M_{\odot}$ ), with a chosen mixing-length parameter $\alpha=2$ and convective overshooting of 0.25 pressure scale heights. The adopted chemical composition is that typical for young stars in the LMC, with $X=0.71$ and $Z=0.01$. The calculation with the spiral-in of the secondary inside the envelope of the primary then creates the initial conditions for the merger phase, which starts when the immersed secondary begins to overfill its Roche lobe.

\section{Results of the simulations and discussion}

The behavior of the merger, or the temporal evolution of the structure of the primary within the secondary orbit depends on how deep and how fast the hydrogen-rich material penetrates into the primary core.

The mass-transfer rate during the merger is determined by the balance between the rate of orbital decay due to tidal and gas/viscous friction on one hand and the rate of orbital expansion due to the mass transfer from the secondary to the primary core on the other hand. At the beginning of the Roche-lobe overflow (RLOF) phase, the mass-loss rate increases sharply. Once the spike in the surface entropy has been removed, the mass-transfer rate first decreases and then slowly increases as the secondary starts to loose the deeper layer, finally achieving a rate of a few solar masses per year. In the particular case shown in Fig. 1a, the final spikes correspond to the nonlinear response of the He shell to the injection of hydrogen-rich material: the He shell itself expands, changing the mass of the primary enclosed within the binary orbit and causing an expansion of the orbit which in turn interrupts the mass transfer temporarily. As can be seen from the figure, the most important events occur only during the last few 

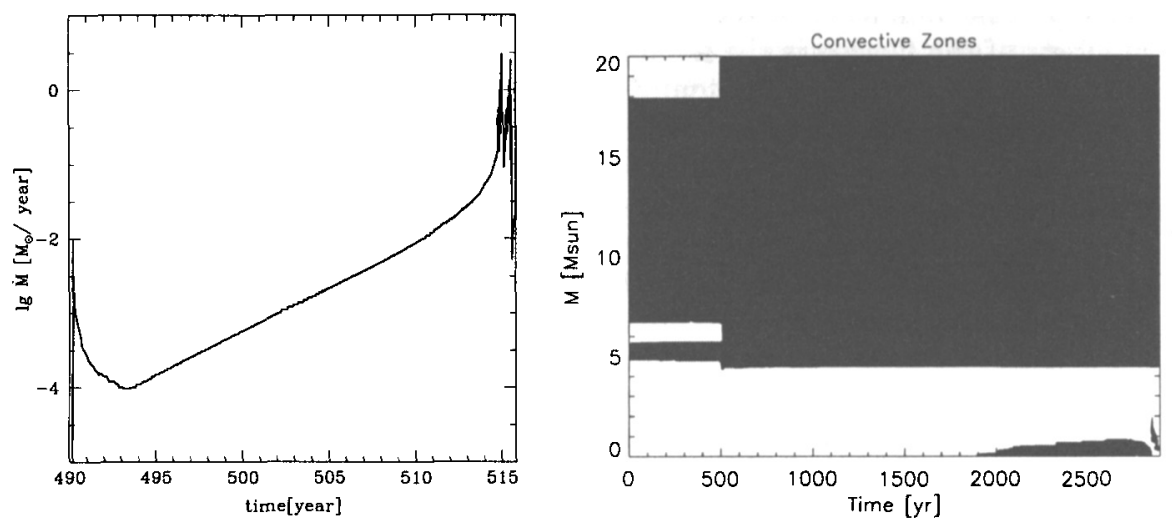

Figure 1. (a) Example for the evolution of mass-transfer rates during the merger; (b) The evolution of convective zones (by mass) since the beginning of the spiral-in.

years, when the mass-loss rate is large and when the entropy of the secondary material is relatively low, so that the stream material is able to penetrate deeply.

Apart from the mass-transfer rate, which is calculated self-consistently, we have varied a number of parameters. The first is the entropy change coefficient which depends on how much the entropy of the material has been changed by the shocks with the ambient medium during the stream infall on to the primary core. This coefficient primarily depends on the initial stream entropy and on the steepness of the pressure and temperature gradients in the region of the primary core and increases as the star climbs up the AGB. Another factor affecting the merger is how much the stream bounces off the core and spreads out its material above the deepest penetration depth. As was shown in IPS, as the core is spun up, the region to which the stream spreads after the interaction with the core decreases with increasing spin rate of the core. A main sequence star of one solar mass of the same age as a secondary of two solar masses has lower entropy. This also affects the stream penetration and therefore the final result of the merger.

We have calculated several models, where we varied these parameters, considering values of $k$ from 0.2 to 0.4 , stream spreading from 0.5 to 2 pressure scale heights and secondaries of 2 and $1 M_{\odot}$. Based on the results of these calculations, we found three different types of merger, which can be distinguished by the strength of the response of the He shell to the merging:

- Explosive mergers (e.g. $18+1 M_{\odot}, k \leq 0.4$ or $18+2 M \odot, k \leq 0.2$ ).

During an explosive merger a large amount of hydrogen is suddenly mixed into the helium-burning shell. This causes a dramatic expansion of the core. As a consequence a large fraction of the core is mixed outwards and the burning convective zone merges with the outer envelope convective zone to form a large single convective zone all the way down to the core. This produces large changes in the surface abundances both during the merger itself and/or during the subsequent delayed dredge-up (see Fig. 1b), where the He abundance is approximately twice solar, and the 
$\mathrm{C}$ abundance may also be significantly enhanced. The explosion can be so effective that it can also lead to the erosion of the carbon-oxygen core. If too much of the $\mathrm{CO}$ core is involved in this explosion event, the core may be destroyed completely.

- Moderate mergers (e.g. $18+2 M_{\odot}, k>0.2$ ).

In a moderately explosive merger only part of the He core expands. In this case the outer convective envelope may dredge up part of the He-rich layer, but an extensive He shell still remains. The merger does not necessarily cause significant changes in the surface composition - the He abundance typically increases by $20-50 \%$.

- Quiet mergers (e.g. $18+2 M_{\odot}, k \approx 0.4$ ).

During this type of merger the material from the secondary only gently touches the outer part of the helium layer which then becomes part of the outer convective envelope. The mass fraction of He in the envelope increases by a few percent only. A narrower stream in this case causes a larger dredge up of $\mathrm{He}$ into the convective envelope.

The classification of the mergers can be well explained by considering the temporal evolution of the convective zones during the merger. As the hydrogen-rich material falls on to the core, it creates a hydrogen-rich zone around it. It is also possible that a convective zone is created in the region where stream material is deposited. On the other hand, the bottom part of the He shell is convective. An explosive event occurs when the hydrogen-rich zone (the stream itself or the hydrogen-rich zone created by the stream) gets into contact with the He convective zone. If at this moment the hydrogen-rich zone contains a large amount of the accumulated hydrogen, an efficient explosion will take place (the timescale for this event is about $0.1-0.5$ year). The creation of the hydrogen convective zone before the hydrogen-rich reservoir gets into contact with the bottom convective zone may lead to the suppression of convection at the bottom of the $\mathrm{He}$ shell. In this case no strong explosion will take place. As a result of such an explosion, part of the dense core is eroded and the temperature drops significantly (see Fig. 2a). The central temperature of the core also drops, increasing the total evolutionary time till the start of carbon core ignition.

The production of s-process elements requires a source of neutrons. The most effective neutron-producing reactions for the temperatures, densities and chemical composition encountered in our problem are $(\alpha, n)$ reactions on the nuclei ${ }^{13} \mathrm{C},{ }^{17} \mathrm{O},{ }^{21} \mathrm{Ne},{ }^{22} \mathrm{Ne}$ and ${ }^{25} \mathrm{Mg}$. In Fig. $2 \mathrm{~b}$ we present a comparison of the cumulative neutron production rates of these 5 reactions in the star before the merger (normal single star) and in the star after the merger (100 years later), for the case of an explosive merger with a well eroded core. It can be seen that the peak in the production rates is about 2 orders of magnitude larger than it was anywhere before the merger, although the region where these processes take place is much smaller by mass (due to the temperature drop near these layers).

After the merger, the star contracts for the first few hundred years and then re-establishes thermal equilibrium. In a more realistic model, the star should experience substantial mass loss in this phase which is currently not included. The reason for the mass loss is the tendency of the star to get rid of the excess angular momentum acquired by the spin-up of the envelope during the spiral-in 

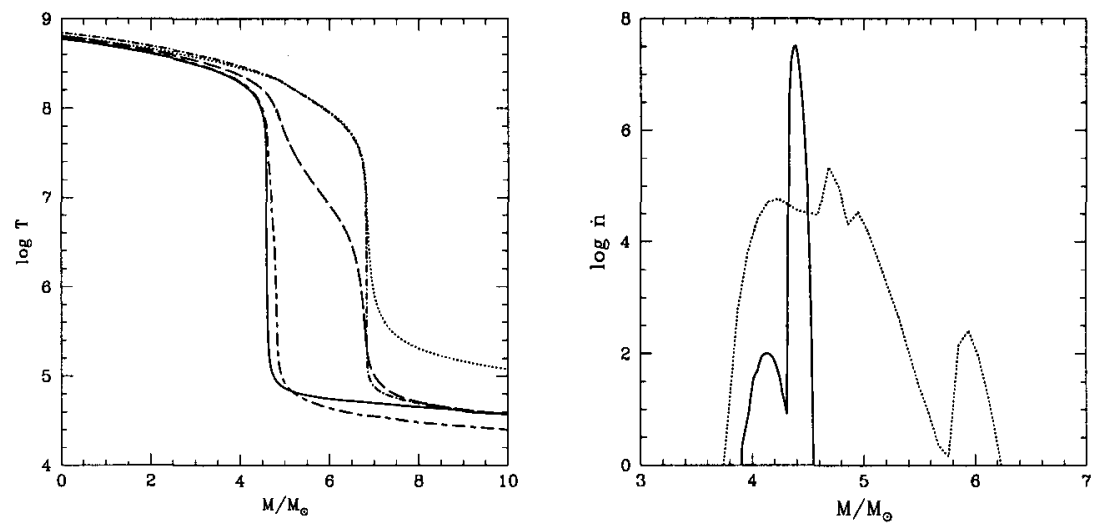

Figure 2. (a) The response of the He shell: temperature of the primary star as a function of mass coordinate at five different stages: at the start of the spiral-in (dotted curve), at the start of the merger (dash-dotted curve), in the middle of the merger when the He shell is expanding (dashed curve), immediately after the He shell has expanded (dash-dashed curve) and 3 years later (solid curve). (b) The accumulated neutron production rates [neutron $\mathrm{g}^{-1} \mathrm{~s}^{-1}$ ], before the merger (dotted curve) and after the merger (solid curve).

phase (mass loss in such a case will not occur in the form of an isotropic wind, but will proceed mainly in the equatorial plane).

The merger scenario considered in this paper produces a star that will ultimately experience a supernova and such a model may represent the progenitor of SN 1987A. Although the models described in this paper cannot explain all the features of the SN 1987A progenitor, the He overabundance in the envelope is close to the observed abundance in the case of SN 1987A. Moreover, substantial s-processing can take place and be much more effective than in the case of a single star. The merged object will be rotating rapidly and form a ring nebula around it with a characteristic dynamical age corresponding to the beginning of the contraction following the merger phase. The models presented here are too evolved to have time to evolve back to the blue region in the H-R diagram before the explosion. This requires slightly less evolved stars at the beginning of the spiral-in phase (Ivanova \& Podsiadlowski 2002, in preparation).

\section{References}

Cannon, R. C. 1993, MNRAS, 263, 817

Iben, I. Jr., \& Livio, M. 1993, PASP, 105, 1373

Hjellming, M. S., \& Taam, R. E. 1991, ApJ, 370, 716

Ivanova, N., Podsiadlowski, P., \& Spruit, H. 2000, in ASP Conf. Ser. Vol. 220, Evolution of Binary and Multiple Star Systems, ed. P. Podsiadlowski, S. Rappaport, A. R. King, F. D'Antona, \& L. Burderi (San Francisco: ASP), 261 
Ivanova, N., Podsiadlowski, P., \& Spruit, H. 2002, MNRAS, in press Meyer, F., \& Meyer-Hofmeister, E. 1979, A\&A, 78, 167 